\title{
ON JOHN DOMAINS IN BANACH SPACES
}

\author{
Y. LI, M. VUORINEN, AND X. WANG *
}

\begin{abstract}
We study the stability of John domains in Banach spaces under removal of a countable set of points. In particular, we prove that the class of John domains is stable in the sense that removing a certain type of closed countable set from the domain yields a new domain which also is a John domain. We apply this result to prove the stability of the inner uniform domains. Finally, we consider a wider class of domains, so called $\psi$-John domains and prove a similar result for this class.
\end{abstract}

\section{INTRODUCTION}

The class of domains, nowadays known as John domains and originally introduced by John [12] in the study of elasticity theory, has been investigated during the past three decades by many people in connection with applications of classical analysis and geometric function theory. See for instance [3, 18, 19] and the references therein. Here we study the classes of John domains and the wider class of $\psi$-John domains [9, 25] and the stability of these two classes of domains under the removal of a countable closed set of points. The motivation for this paper stems from the discussions in [10, 24], where the effect of the removal of a finite set of points was examined. See also the very recent paper [14].

Suppose that $D$ is a domain in a real Banach space $E$ with dimension at least 2 and let $P_{D}$ denote a countable set in $D$ such that the quasihyperbolic distance w.r.t. $D$ between each pair of distinct points in $P_{D}$ is at least $b$ where $b>0$ is a constant. The first main result of this paper shows that $D$ is a $c$-John domain if and only if $D \backslash P_{D}$ is a $c_{1}$-John domain, where $c$ and $c_{1}$ are two constants depending only on each other and on $b$. Applying this result, we show that $D$ is inner uniform if and only if $D \backslash P_{D}$ is inner uniform. Our second main result shows that $D$ is a $\psi$-John domain if and only if $D \backslash P_{D}$ is a $\psi_{1^{-}}$John domain, where $\psi$ and $\psi_{1}$ depend only on each other and on $b$.

The methods applied in the proofs rely on standard notions of metric space theory: curves, their lengths, and nearly length-minimizing curves. It should be noted that we employ several metric space structures on the domain $D$ including hyperbolic

File: john130923.tex, printed: 2022-3-22, 8.08

2010 Mathematics Subject Classification. Primary: 30C65, 30F45; Secondary: 30C20.

Key words and phrases. removability, John domain, inner uniform domain, quasihyperbolic metric.

* Corresponding author

This research was supported by the Academy of Finland, Project 2600066611, and by NSF of China (No. 11071063). 
type metrics. We use three metrics: the norm metric, the distance ratio metric and the quasihyperbolic metric on the domain $D$ and, moreover, also on its subdomains.

\section{THE SECOND SECTION}

Throughout the paper, we always assume that $E$ denotes a real Banach space with dimension at least 2 . The norm of a vector $z$ in $E$ is written as $|z|$, and for each pair of points $z_{1}, z_{2}$ in $E$, the distance between them is denoted by $\left|z_{1}-z_{2}\right|$, the closed line segment with endpoints $z_{1}$ and $z_{2}$ by $\left[z_{1}, z_{2}\right]$. We always use $\mathbb{B}\left(x_{0}, r\right)$ to denote the open ball $\left\{x \in E:\left|x-x_{0}\right|<r\right\}$ centered at $x_{0}$ with radius $r>0$. Similarly, for the closed balls and spheres, we use the usual notations $\overline{\mathbb{B}}\left(x_{0}, r\right)$ and $\mathbb{S}\left(x_{0}, r\right)$, respectively.

For each pair of points $z_{1}, z_{2}$ in $D$, the distance ratio metric $j_{D}\left(z_{1}, z_{2}\right)$ between $z_{1}$ and $z_{2}$ is defined by

$$
j_{D}\left(z_{1}, z_{2}\right)=\log \left(1+\frac{\left|z_{1}-z_{2}\right|}{\min \left\{d_{D}\left(z_{1}\right), d_{D}\left(z_{2}\right)\right\}}\right),
$$

where $d_{D}(z)$ denotes the distance from $z$ to the boundary $\partial D$ of $D$.

The quasihyperbolic length of a rectifiable arc or a path $\gamma$ in $D$ is the number (cf. $[1,5,6,22])$

$$
\ell_{k}(\gamma)=\int_{\gamma} \frac{1}{d_{D}(z)}|d z|
$$

For each pair of points $z_{1}, z_{2}$ in $D$, the quasihyperbolic distance $k_{D}\left(z_{1}, z_{2}\right)$ between $z_{1}$ and $z_{2}$ is defined in the usual way:

$$
k_{D}\left(z_{1}, z_{2}\right)=\inf \ell_{k}(\alpha),
$$

where the infimum is taken over all rectifiable $\operatorname{arcs} \alpha$ joining $z_{1}$ to $z_{2}$ in $D$.

For all $z_{1}, z_{2}$ in $D$, we have (cf. [22])

$$
\begin{gathered}
k_{D}\left(z_{1}, z_{2}\right) \geq \inf \left\{\log \left(1+\frac{\ell(\alpha)}{\min \left\{d_{D}\left(z_{1}\right), d_{D}\left(z_{2}\right)\right\}}\right)\right\} \geq j_{D}\left(z_{1}, z_{2}\right) \\
\geq\left|\log \frac{d_{D}\left(z_{2}\right)}{d_{D}\left(z_{1}\right)}\right|,
\end{gathered}
$$

where the infimum is taken over all rectifiable curves $\alpha$ in $D$ connecting $z_{1}$ and $z_{2}$, $\ell(\alpha)$ denotes the length of $\alpha$. Next, if $\left|z_{1}-z_{2}\right|<d_{D}\left(z_{1}\right)$, then we have [22], [28, Lemma 2.11]

$$
k_{D}\left(z_{1}, z_{2}\right) \leq \log \left(1+\frac{\left|z_{1}-z_{2}\right|}{d_{D}\left(z_{1}\right)-\left|z_{1}-z_{2}\right|}\right) \leq \frac{\left|z_{1}-z_{2}\right|}{d_{D}\left(z_{1}\right)-\left|z_{1}-z_{2}\right|},
$$

where the last inequality follows from the following elementary inequality

$$
\frac{r}{1-r / 2} \leq \log \frac{1}{1-r} \leq \frac{r}{1-r} \quad \text { for } 0 \leq r<1 .
$$

Gehring and Palka [5] introduced the quasihyperbolic metric of a domain in $R^{n}$. Many of the basic properties of this metric may be found in [6, 11, 13, 15, 20, 27. Recall that an arc $\alpha$ from $z_{1}$ to $z_{2}$ is a quasihyperbolic geodesic if $\ell_{k}(\alpha)=k_{D}\left(z_{1}, z_{2}\right)$. 
Each subarc of a quasihyperbolic geodesic is obviously a quasihyperbolic geodesic. It is known that a quasihyperbolic geodesic between every pair of points in $E$ exists if the dimension of $E$ is finite, see [6, Lemma 1]. This is not true in infinite dimensional Banach spaces (cf. [22, Example 2.9]). In order to remedy this shortage, Väisälä introduced the following concepts [24].

Definition 2.3. Let $D \neq E$ and $c \geq 1$. An arc $\alpha \subset D$ is a $c$-neargeodesic if $\ell_{k}(\alpha[x, y]) \leq c k_{D}(x, y)$ for all $x, y \in \alpha$.

In [24], Väisälä proved the following property concerning the existence of neargeodesics in $E$.

Lemma 2.4. ([24, Theorem 3.3]) Let $z_{1}, z_{2} \in D \neq E$ and let $c>1$. Then there is a c-neargeodesic joining $z_{1}$ and $z_{2}$ in $D$.

Definition 2.5. A domain $D$ in $E$ is called $c$-John domain in the norm metric provided there exists a constant $c$ with the property that each pair of points $z_{1}, z_{2}$ in $D$ can be joined by a rectifiable arc $\alpha$ in $D$ such that for all $z \in \alpha$ the following holds:

$$
\min \left\{\ell\left(\alpha\left[z_{1}, z\right]\right), \ell\left(\alpha\left[z_{2}, z\right]\right)\right\} \leq c d_{D}(z),
$$

where $\alpha\left[z_{j}, z\right]$ denotes the part of $\alpha$ between $z_{j}$ and $z$ (cf. [3, 19]). The arc $\alpha$ is called to be a c-cone arc .

A domain $D$ in $E$ is said to be a c-uniform domain (cf. [17, 18, 21, 24, 26]) if there is a constant $c \geq 1$ such that each pair of points $z_{1}, z_{2} \in D$ can be joined by an arc $\alpha$ satisfying $(2.6)$ and

$$
\ell(\alpha) \leq c\left|z_{1}-z_{2}\right|
$$

We also say that $\alpha$ is a $c$-uniform arc (cf. [25]).

For $z_{1}, z_{2} \in D$, the inner length metric $\lambda_{D}\left(z_{1}, z_{2}\right)$ between these points is defined by

$$
\lambda_{D}\left(z_{1}, z_{2}\right)=\inf \left\{\ell(\alpha): \alpha \subset D \text { is a rectifiable arc joining } z_{1} \text { and } z_{2}\right\} .
$$

We say that a domain $D$ in $E$ is an inner c-uniform domain if there is a constant $c \geq 1$ such that each pair of points $z_{1}, z_{2} \in D$ can be joined by an arc $\alpha$ satisfying (2.6) and

$$
\ell(\alpha) \leq c \lambda_{D}\left(z_{1}, z_{2}\right) .
$$

Such an arc $\alpha$ is called to be an inner c-uniform arc (cf. [25]).

Obviously, uniform domains are inner uniform domains, but inner uniform does not imply uniform. See [2, 4, 6, 17, 18, 21, 24, 25] for more details on uniform domains and inner uniform domains.

Remarks. If we replace (2.6), 2.7) and (2.8) by

$$
\begin{gathered}
\min \left\{\operatorname{diam}\left(\alpha\left[z_{1}, z\right]\right), \operatorname{diam}\left(\alpha\left[z_{2}, z\right]\right)\right\} \leq c d_{D}(z), \\
\operatorname{diam}(\alpha) \leq c\left|z_{1}-z_{2}\right|
\end{gathered}
$$

and

$$
\operatorname{diam}(\alpha) \leq c \lambda_{D}\left(z_{1}, z_{2}\right)
$$


then we get concepts which in the case $E=\mathbb{R}^{n}$ are $n$-quantitatively equivalent to $c$-John domain, $c$-uniform domain and inner $c$-uniform domain, respectively [19]. But in an arbitrary Banach space, each of these three conditions leads to a wider class of domains. For example, the broken tube domain considered by Väisälä [22, 4.12] (see also [23]) is neither John nor quasiconvex (a metric space is c-quasiconvex if each pair of points $x, y$ can be joined by an arc with (2.7) holds). Nevertheless, one can join a given pair of points in this bounded domain by arcs satisfying (2.9), (2.10) and (2.11).

Various classes of domains have been studied in analysis (e.g. see [7]). For some classes, the removal of a finite number of points from a domain may yield a domain no longer in this class [7]. In [10, the authors proved that the removal of a finite number of points from a John domain yields another John domain.

Lemma 2.12. ([10, Theorem 1.4]) A domain $D \subset \mathbb{R}^{n}$ is a John domain if and only if $G=D \backslash P$ is also a John domain, where $P=\left\{p_{1}, p_{2}, \cdots, p_{m}\right\} \subset D$.

In general, when $P$ is an infinite closed set in $D, D \backslash P$ need not be a John domain ([10, Example 1.5]). In this paper, we continue the study of the removability properties of John domains and prove that if $P$ satisfies a certain separation condition, then $D \backslash P$ is still a John domain if $D$ is a John domain.

Let $b>0$ be a constant. In what follows, for a domain $D$ in $E$, and for a sequence $X=\left\{x_{j}: j=1,2, \ldots\right\}$ of points in $D$ satisfying the quasihyperbolic separation condition

$$
k_{D}\left(x_{i}, x_{j}\right) \geq b \text { for } i \neq j
$$

we always write

$$
P_{D}=X
$$

Further, we assume that the set $P_{D}$ satisfying the quasihyperbolic separation condition contains at least two points, and in the following, without loss of generality, we may assume that $b=\frac{1}{2}$.

Given $x \in D$ and $s \in(0,1)$, for $z_{1}, z_{2} \in \mathbb{B}\left(x, s d_{D}(x)\right)$, we see from 2.2$)$ that

$$
k_{D}\left(z_{1}, z_{2}\right) \leq 2 \log (1 /(1-s)) .
$$

This fact, together with the definition of $P_{D}$, yields the following lemma.

Lemma 2.13. For all $w \in D$, there exists at most one point $x_{i}$ of $P_{D}$ such that $x_{i} \in \mathbb{B}\left(w, \frac{1}{6} d_{D}(w)\right)$.

Lemma 2.14. ([24, Lemma 6.7]) Suppose that $G$ is a c-uniform domain and that $x_{0} \in G$. Then $G_{0}=G \backslash\left\{x_{0}\right\}$ is $c_{1}$-uniform with $c_{1}=c_{1}(c)$ (This means that $c_{1}$ is a constant depending only on c). Moreover, from the proof of [24, Lemma 6.7] we see that $c_{1} \leq 9 c$.

We note that each ball $\mathbb{B}(x, r)$ is 2 -uniform and $\mathbb{B}(x, r) \backslash\{x\}$ is 10 -uniform by the proof of [24, Theorem 6.5]. By Lemma 2.13 and 2.14, the following holds.

Lemma 2.15. For $x_{0} \in D, \mathbb{B}\left(x_{0}, \frac{1}{6} d_{D}\left(x_{0}\right)\right) \backslash P_{D}$ is $c_{2}$-uniform with $2 \leq c_{2} \leq 18$. 
Lemma 2.16. lemFor $x, y \in D$, if there is a $c_{3}$-cone arc $\gamma$ joining $x, y$ in $D$, then for each $w \in \gamma$ the following holds:

$$
d_{D}(w) \geq \frac{1}{2 c_{3}} \min \left\{d_{D}(x), d_{D}(y)\right\} .
$$

Moreover, if $\ell(\gamma[x, w]) \leq \ell(\gamma[y, w])$, then

$$
d_{D}(w) \geq \frac{1}{2 c_{3}} d_{D}(x) .
$$

Otherwise,

$$
d_{D}(w) \geq \frac{1}{2 c_{3}} d_{D}(y) .
$$

Proof. Let $w_{0} \in \gamma$ bisect the arclength of $\gamma$. Obviously, we only need to consider the case $w \in \gamma\left[x, w_{0}\right]$ since the discussion for the case $w \in \gamma\left[y, w_{0}\right]$ is similar.

If $\ell(\gamma[x, w]) \leq \frac{1}{2} d_{D}(x)$, then

$$
d_{D}(w) \geq d_{D}(x)-\ell(\gamma[x, w]) \geq \frac{1}{2} d_{D}(x) .
$$

If $\ell(\gamma[x, w])>\frac{1}{2} d_{D}(x)$, then we have

$$
d_{D}(w) \geq \frac{1}{c_{3}} \ell(\gamma[x, w])>\frac{1}{2 c_{3}} d_{D}(x) .
$$

The proof is complete.

Let us recall the following result from [16].

Lemma 2.17. (16, Theorem 1.2]) Suppose that $D_{1}$ and $D_{2}$ are convex domains in $E$, where $D_{1}$ is bounded and $D_{2}$ is c-uniform, and that there exist $z_{0} \in D_{1} \cap D_{2}$ and $r>0$ such that $\mathbb{B}\left(z_{0}, r\right) \subset D_{1} \cap D_{2}$. If there exist $R_{1}>0$ and a constant $c_{0}>0$ such that $R_{1} \leq c_{0} r$ and $D_{1} \subset \overline{\mathbb{B}}\left(z_{0}, R_{1}\right)$, then $D_{1} \cup D_{2}$ is a $c^{\prime}$-uniform domain with $c^{\prime}=\frac{1}{2}(c+1)\left(6 c_{0}+1\right)+c$.

By Lemma 2.13, 2.4 and 2.17, we easily have the following lemma.

Lemma 2.18. Let $D \subset E$ be a domain. For $y_{1}, w_{1} \in D$, if

$$
\mathbb{B}\left(y_{1}, \frac{1}{32} d_{D}\left(y_{1}\right)\right) \cap \mathbb{B}\left(w_{1}, \frac{1}{32} d_{D}\left(w_{1}\right)\right) \neq \emptyset,
$$

then $D_{0} \backslash P_{D}$ is a $660 c_{2}^{2}$-uniform domain, where $D_{0}=\mathbb{B}\left(y_{1}, \frac{1}{16} d_{D}\left(y_{1}\right)\right) \cup \mathbb{B}\left(w_{1}, \frac{1}{32} d_{D}\left(w_{1}\right)\right)$.

\section{Stability OF John DOMAins AND AN APplication}

Before the formulation of our main theorem, we prove a key lemma.

Lemma 3.1. Let $D \subset E$ be a domain. For $z_{1}, z_{2} \in G=D \backslash P_{D}$, let $\gamma$ be a rectifiable arc joining $z_{1}$ and $z_{2}$ in $D$. Then there exists an arc $\alpha \subset G$ joining $z_{1}$ and $z_{2}$ such that $\ell(\alpha) \leq 660 c_{2}^{2} \ell(\gamma)$. Moreover, if $\gamma$ is a c-cone arc in $D$, then $\alpha$ is $a\left(2^{18} c c_{2}^{3}+660 c_{2}^{2}\right)$-cone arc in $G$, where $c>1$ is a constant and $c_{2}$ is the constant from Lemma 2.15 . 
Proof. For given $z_{1}$ and $z_{2}$ in $G$, let $\gamma$ be a rectifiable arc joining $z_{1}$ and $z_{2}$ in $D$ and let

$$
U=\left\{u \in \gamma: d_{D}(u)>64 d_{G}(u)\right\} .
$$

If $U=\emptyset$, then let $\alpha_{0}=\gamma$. Obviously, Lemma 3.1 holds.

In the following, we assume that $U \neq \emptyset$. We prove this lemma by considering three cases.

Case 3.2. There exists some point $w_{0} \in \gamma$ such that $\left\{z_{1}, z_{2}\right\} \subset \overline{\mathbb{B}}\left(w_{0}, \frac{1}{32} d_{D}\left(w_{0}\right)\right)$.

Then by Lemma 2.15, we know that there is a $c_{2}$-uniform arc $\alpha_{1}$ joining $z_{1}$ and $z_{2}$ in $G$ which is the desired since

$$
\ell\left(\alpha_{1}\right) \leq c_{2}\left|z_{1}-z_{2}\right| \leq c_{2} \ell(\gamma) .
$$

Let $z_{0} \in \gamma$ be a point such that $\ell\left(\gamma\left[z_{1}, z_{0}\right]\right)=\ell\left(\gamma\left[z_{2}, z_{0}\right]\right)$.

Case 3.3. For all $w \in \gamma,\left\{z_{1}, z_{2}\right\} \nsubseteq \overline{\mathbb{B}}\left(w, \frac{1}{32} d_{D}(w)\right)$, but there is a point $w_{1} \in$ $\gamma\left[z_{1}, z_{0}\right]$ such that $z_{2} \in \overline{\mathbb{B}}\left(w_{1}, \frac{1}{32} d_{D}\left(w_{1}\right)\right)$ or a point $w_{2} \in \gamma\left[z_{2}, z_{0}\right]$ such that $z_{1} \in$ $\overline{\mathbb{B}}\left(w_{2}, \frac{1}{32} d_{D}\left(w_{2}\right)\right)$.

Obviously, we only need to consider the former case since the discussion for the latter case is similar. Without loss of generality, we may assume that $w_{1}$ is the first point in $\gamma\left[z_{1}, z_{0}\right]$ along the direction from $z_{1}$ to $z_{0}$ such that $z_{2} \in \overline{\mathbb{B}}\left(w_{1}, \frac{1}{32} d_{D}\left(w_{1}\right)\right)$.

Subcase 3.4. $U \cap \gamma\left[z_{1}, w_{1}\right]=\emptyset$.

That is, for all $w \in \gamma\left[z_{1}, w_{1}\right], d_{D}(w) \leq 64 d_{G}(w)$. By Lemma 2.15, there exists a $c_{2^{-}}$ uniform arc $\eta_{1}$ joining $w_{1}$ and $z_{2}$ in $G$. Then we come to prove that $\alpha_{2}=\gamma\left[z_{1}, w_{1}\right] \cup \eta_{1}$ is the desired arc. By the choice of $\eta_{1}$, we know that

$$
\ell\left(\alpha_{2}\right) \leq c_{2}\left|w_{1}-z_{2}\right|+\ell\left(\gamma\left[z_{1}, w_{1}\right]\right) \leq c_{2} \ell(\gamma) \text {. }
$$

Assume further that $\gamma$ is a $c$-cone arc. Then we let $u_{0}$ bisect the arclength of $\eta_{1}$. If $w \in \gamma\left[z_{1}, w_{1}\right]$, then

$$
\ell\left(\alpha_{2}\left[z_{1}, w\right]\right)=\ell\left(\gamma\left[z_{1}, w\right]\right) \leq c d_{D}(w) \leq 64 c d_{G}(w) .
$$

If $w \in \eta_{1}\left[w_{1}, u_{0}\right]$, then Lemma 2.16 yields

$\ell\left(\alpha_{2}\left[z_{1}, w\right]\right)=\ell\left(\gamma\left[z_{1}, w_{1}\right]\right)+\ell\left(\eta_{1}\left[w_{1}, w\right]\right) \leq 64 c d_{G}\left(w_{1}\right)+c_{2} d_{G}(w) \leq(128 c+1) c_{2} d_{G}(w)$.

If $w \in \eta_{1}\left[u_{0}, z_{2}\right]$, then

$$
\ell\left(\alpha\left[z_{2}, w\right]\right)=\ell\left(\eta_{1}\left[z_{2}, w\right]\right) \leq c_{2} d_{G}(w) .
$$

Hence $\alpha_{2}$ is the desired.

Subcase 3.5. $U \cap \gamma\left[z_{1}, w_{1}\right] \neq \emptyset$.

If $z_{1} \in U$, then let $y_{1}=z_{1}$. Otherwise, let $y_{1}$ be the first point in $\gamma\left[z_{1}, w_{1}\right]$ along the direction from $z_{1}$ to $w_{1}$ such that

$$
d_{D}\left(y_{1}\right)=64 d_{G}\left(y_{1}\right) .
$$

We first consider the case:

$$
\mathbb{B}\left(y_{1}, \frac{1}{32} d_{D}\left(y_{1}\right)\right) \cap \mathbb{B}\left(w_{1}, \frac{1}{32} d_{D}\left(w_{1}\right)\right) \neq \emptyset .
$$


By Lemma 2.18, we know that there is a $660 c_{2}^{2}$-uniform arc $\eta_{2}$ joining $y_{1}$ and $z_{2}$ in $G$, then let $\alpha_{3}=\gamma\left[z_{1}, y_{1}\right] \cup \eta_{2}$. Here and in the following, we assume that $\gamma\left[z_{1}, y_{1}\right]=\left\{z_{1}\right\}$ if $z_{1}=y_{1}$.

If $y_{1}=z_{1}$, then $\alpha_{3}=\eta_{2}$, and obviously, it satisfies Lemma 3.1. If $y_{1} \neq z_{1}$, then replacing $c_{2}$ by $660 c_{2}^{2}$, similar arguments as in Subcase 3.4 show that $\alpha_{3}$ is the desired.

In the following, we assume

$$
\mathbb{B}\left(y_{1}, \frac{1}{32} d_{D}\left(y_{1}\right)\right) \cap \mathbb{B}\left(w_{1}, \frac{1}{32} d_{D}\left(w_{1}\right)\right)=\emptyset
$$

and we come to construct an arc $\alpha_{4}$ satisfying the lemma. We first show the following claim.

Claim 3.7. There exists a sequence of points $\left\{y_{i}\right\}_{i=1}^{p_{1}}$ in $\gamma$, where $p_{1} \geq 3$ is an odd number, satisfying the following conditions.

(1) $y_{1}=z_{1}$ or $y_{1}$ is first point in $\gamma\left[z_{1}, w_{1}\right]$ from $z_{1}$ to $w_{1}$ such that $d_{D}\left(y_{1}\right)=$ $64 d_{G}\left(y_{1}\right)$

(2) For each even number $j \in\left\{1,2, \ldots, p_{1}\right\}, d_{G}\left(y_{j}\right) \geq \frac{1}{66} d_{D}\left(y_{j}\right)$ and $d_{D}\left(y_{p_{1}}\right) \leq$ $128 d_{G}\left(y_{p_{1}}\right)$

(3) If $p_{1} \geq 5$, then for each even number $j \in\left\{1,2, \ldots, p_{1}-2\right\}, y_{j+1}$ is the first point in $\gamma\left[y_{j}, w_{1}\right]$ from $y_{j}$ to $w_{1}$ such that $d_{D}\left(y_{j+1}\right)=128 d_{G}\left(y_{j+1}\right)$;

(4) $p_{1}$ is the smallest integer with $y_{p_{1}} \in \mathbb{S}\left(w_{1}, \frac{1}{32} d_{D}\left(w_{1}\right)\right)$ or $\mathbb{B}\left(y_{p_{1}}, \frac{1}{32} d_{D}\left(y_{p_{1}}\right)\right) \cap$ $\mathbb{B}\left(w_{1}, \frac{1}{32} d_{D}\left(w_{1}\right)\right) \neq \emptyset$ (see Figures 1 and 2$)$.

For a proof, we let $y_{2} \in \gamma\left[y_{1}, w_{1}\right] \cap \mathbb{S}\left(y_{1}, \frac{1}{32} d_{D}\left(y_{1}\right)\right.$ be such that

$$
\gamma\left(y_{2}, w_{1}\right] \cap \mathbb{S}\left(y_{1}, \frac{1}{32} d_{D}\left(y_{1}\right)\right)=\emptyset
$$

where $\gamma\left(y_{2}, w_{1}\right]$ denote the part $\gamma$ from $y_{2}$ to $w_{1}$ such that $y_{2} \notin \gamma\left[y_{2}, w_{1}\right]$. Then

$$
d_{D}\left(y_{2}\right) \leq d_{D}\left(y_{1}\right)+\left|y_{1}-y_{2}\right|=\frac{33}{32} d_{D}\left(y_{1}\right) .
$$

By Lemma 2.13 and $(3.6)$, we know that there exists one and only one point, say $x_{s}$, in $\mathbb{B}\left(y_{1}, \frac{1}{6} d_{D}\left(y_{1}\right)\right) \cap P_{D}$, and so

$$
d_{G}\left(y_{2}\right)=\left|y_{2}-x_{s}\right| \geq\left|y_{2}-y_{1}\right|-\left|y_{1}-x_{s}\right| \geq \frac{1}{64} d_{D}\left(y_{1}\right),
$$

which, together with (3.8), shows that

$$
d_{G}\left(y_{2}\right) \geq \frac{1}{66} d_{D}\left(y_{2}\right)
$$

If for all $w \in \gamma\left[y_{2}, w_{1}\right] \backslash \mathbb{B}\left(w_{1}, \frac{1}{32} d_{D}\left(w_{1}\right)\right), d_{D}(w) \leq 128 d_{G}(w)$, then the claim obviously holds by letting $y_{3} \in \gamma\left[y_{2}, w_{1}\right] \cap \mathbb{S}\left(w_{1}, \frac{1}{32} d_{D}\left(w_{1}\right)\right.$ and $p_{1}=3$.

If there is some $w_{0} \in \gamma\left[y_{2}, w_{1}\right] \backslash \mathbb{B}\left(w_{1}, \frac{1}{32} d_{D}\left(w_{1}\right)\right.$ such that $d_{D}\left(w_{0}\right)>128 d_{G}\left(w_{0}\right)$, then let $y_{3}$ be the first point in $\gamma\left[y_{2}, w_{1}\right]$ from $y_{2}$ to $w_{1}$ such that $d_{D}\left(y_{3}\right)=128 d_{G}\left(y_{3}\right)$. 


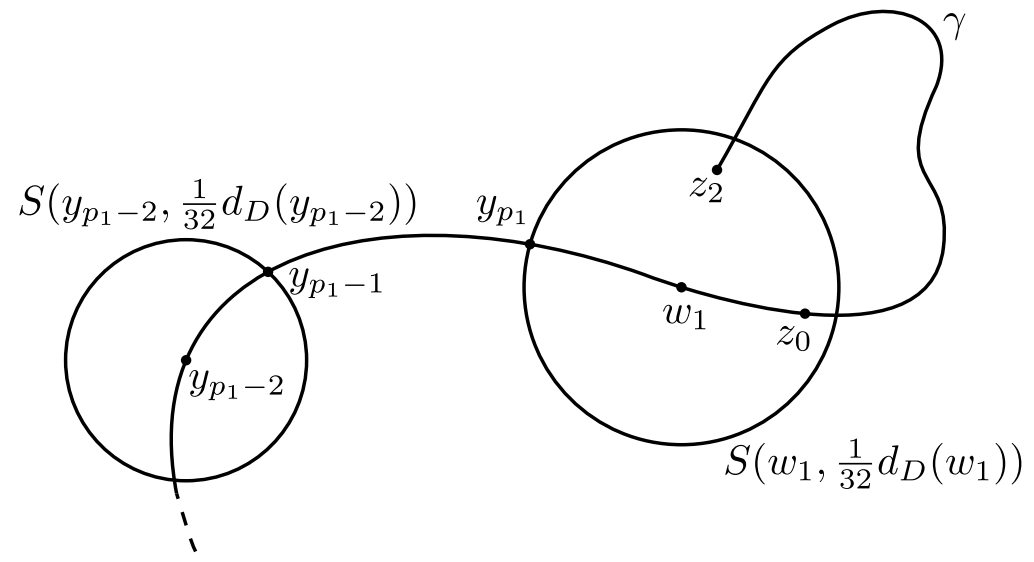

FiguRE 1. For all $w \in \gamma\left[y_{p_{1}-1}, w_{1}\right] \backslash \mathbb{B}\left(w_{1}, \frac{1}{32} d_{D}\left(w_{1}\right)\right), d_{D}(w) \leq 128 d_{G}(w)$

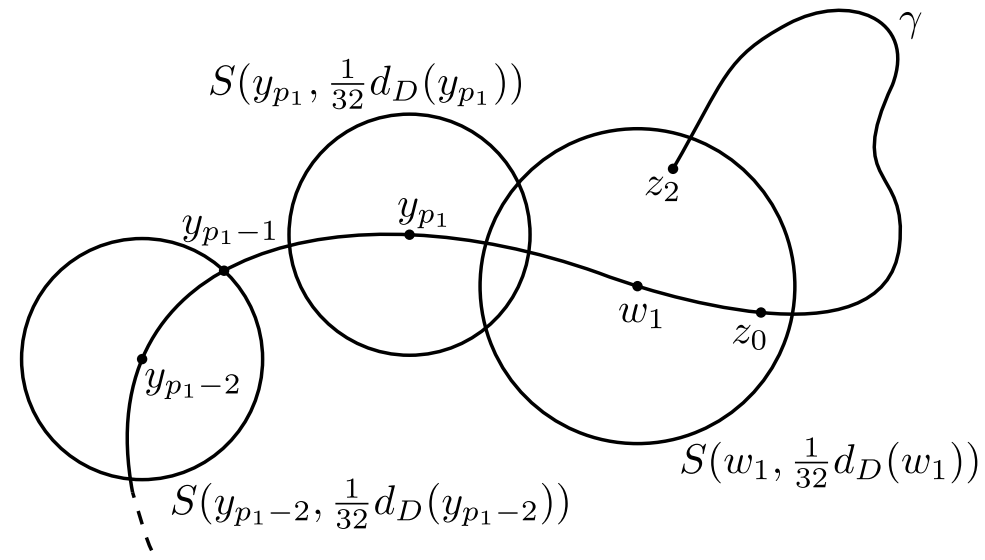

Figure 2. $\mathbb{B}\left(y_{p_{1}}, \frac{1}{32} d_{D}\left(y_{p_{1}}\right)\right) \cap \mathbb{B}\left(w_{1}, \frac{1}{32} d_{D}\left(w_{1}\right)\right) \neq \emptyset$

If $\mathbb{B}\left(y_{3}, \frac{1}{32} d_{D}\left(y_{3}\right)\right) \cap \mathbb{B}\left(w_{1}, \frac{1}{32} d_{D}\left(w_{1}\right)\right) \neq \emptyset$, then the claim holds and $p_{1}=3$. Otherwise, let $y_{4} \in \gamma\left[y_{3}, w_{1}\right] \cap \mathbb{S}\left(y_{3}, \frac{1}{32} d_{D}\left(y_{3}\right)\right.$ be such that

$$
\gamma\left(y_{4}, w_{1}\right] \cap \mathbb{S}\left(y_{3}, \frac{1}{32} d_{D}\left(y_{3}\right)\right)=\emptyset
$$

Then by Lemma 2.13, and a similar argument as in the proof of (3.9), we have

$$
d_{G}\left(y_{4}\right) \geq \frac{1}{66} d_{D}\left(y_{4}\right)
$$

If for all $w \in \gamma\left[y_{4}, w_{1}\right] \backslash \mathbb{B}\left(w_{1}, \frac{1}{32} d_{D}\left(w_{1}\right)\right), d_{D}(w) \leq 128 d_{G}(w)$, then we complete the proof of the claim by letting $y_{5} \in \gamma\left[y_{4}, w_{1}\right] \cap \mathbb{S}\left(w_{1}, \frac{1}{32} d_{D}\left(w_{1}\right) . \cdots\right.$. 
By repeating this process for finite steps, we get a sequence $\left\{y_{i}\right\}_{i=1}^{p_{1}} \in \gamma$ satisfying Claim 3.7, where $p_{1}<\frac{M}{\log \frac{33}{32}}$, since for each $i \in\left\{1,2, \ldots, \frac{p_{1}-1}{2}\right\}$,

$$
\ell_{k_{D}}\left(\gamma\left[y_{2 i-1}, y_{2 i}\right]\right) \geq \log \left(1+\frac{\left|y_{2 i-1}-y_{2 i}\right|}{d_{D}\left(y_{2 i-1}\right)}\right)=\log \frac{33}{32},
$$

and $M=\ell_{k_{D}}\left(\gamma\left[z_{1}, z_{2}\right]\right)$. Hence Claim 3.7 holds.

We continue the construction of $\alpha_{4}$. Let $\gamma_{1}=\gamma\left[z_{1}, y_{1}\right]$ and for each $j \in\left\{2, \ldots, \frac{p_{1}+1}{2}\right\}$, let $\gamma_{j}=\gamma\left[y_{2 j-2}, y_{2 j-1}\right]$. By Lemma 2.15, we know that for each $j \in\left\{1,2, \ldots, \frac{p_{1}-1}{2}\right\}$, there exists a $c_{2}$-uniform arc $\beta_{j} \subset G$ joining $y_{2 j-1}$ and $y_{2 j}$. By Lemmas 2.15 and 2.18, there exists a $660 c_{2}^{2}$-uniform arc $\eta_{3}$ joining $y_{p_{1}}$ and $z_{2}$. Take

$$
\alpha_{4}=\gamma_{1} \cup \beta_{1} \cup \gamma_{2} \cup \ldots \cup \beta_{\frac{p_{1}-1}{2}} \cup \gamma_{\frac{p_{1}+1}{2}} \cup \eta_{3} \text {. }
$$

Now, we come to show that $\alpha_{4}$ is the desired arc.

First observe that

$$
\ell\left(\alpha_{4}\right) \leq 660 c_{2}^{2} \ell(\gamma)
$$

To prove that $\alpha_{4}$ is a cone arc in $G$, it is enough to show that

$$
\min \left\{\ell\left(\alpha_{4}\left[z_{1}, w\right]\right), \ell\left(\alpha_{4}\left[w, z_{2}\right]\right)\right\} \leq\left(2^{18} c c_{2}^{3}+660 c_{2}^{2}\right) d_{G}(w) .
$$

If $w \in \gamma_{1} \cup \gamma_{2} \cup \ldots \cup \gamma_{\frac{p_{1}+1}{2}}$, then from the assumption, Claim 3.7 and 3.10), the above inequality obviously holds.

For the case where $w \in \beta_{1} \cup \ldots \cup \beta_{\frac{p_{1}-1}{2}}$, we see that there exists some $i \in$ $\left\{1,2, \ldots, \frac{p_{1}-1}{2}\right\}$ such that $w \in \beta_{i}$. By Claim 3.7.

$$
d_{G}\left(y_{2 i}\right) \geq \frac{1}{66} d_{D}\left(y_{2 i}\right) \geq \frac{1}{66}\left(d_{D}\left(y_{2 i-1}\right)-\left|y_{2 i-1}-y_{2 i}\right|\right) \geq \frac{62}{33} d_{G}\left(y_{2 i-1}\right),
$$

whence Lemma 2.16 yields

$$
d_{G}(w) \geq \frac{1}{2 c_{2}} \min \left\{d_{G}\left(y_{2 i-1}\right), d_{G}\left(y_{2 i}\right)\right\}=\frac{1}{2 c_{2}} d_{G}\left(y_{2 i-1}\right),
$$

which, together with Claim 3.7, leads to

$$
\begin{aligned}
\ell\left(\alpha_{4}\left[z_{1}, w\right]\right) & \leq c_{2} \ell\left(\gamma\left[z_{1}, y_{2 i-1}\right]\right)+c_{2}\left|y_{2 i-1}-y_{2 i}\right| \\
& \leq 4 c_{2}(32 c+1) d_{G}\left(y_{2 i-1}\right) \leq 8 c_{2}^{2}(32 c+1) d_{G}(w) .
\end{aligned}
$$

For the remaining case where $w \in \eta_{3}$, we let $u_{0}$ bisect the arclength of $\eta_{3}$. If $w \in \eta_{3}\left[z_{2}, u_{0}\right]$, then, obviously,

$$
\ell\left(\alpha_{4}\left[z_{2}, w\right]\right) \leq c_{2} d_{G}(w) .
$$

If $w \in \eta_{3}\left[y_{3}, u_{0}\right]$, then by Lemma 2.16 and Claim 3.7, we have

$$
\begin{aligned}
\ell\left(\alpha_{4}\left[z_{1}, w\right]\right) & \leq c_{2} \ell\left(\gamma\left[z_{1}, y_{p_{1}}\right]\right)+\ell\left(\eta_{3}\left[y_{p_{1}}, w\right]\right) \\
& \leq 128 c c_{2} d_{G}\left(y_{p_{1}}\right)+660 c_{2}^{2} d_{G}(w) \leq\left(2^{18} c c_{2}^{3}+660 c_{2}^{2}\right) d_{G}(w) .
\end{aligned}
$$

Case 3.12. $z_{1} \notin \bigcup_{w \in \gamma\left[z_{2}, z_{0}\right]} \overline{\mathbb{B}}\left(w, \frac{1}{32} d_{D}(w)\right)$ and $z_{2} \notin \bigcup_{w \in \gamma\left[z_{1}, z_{0}\right]} \overline{\mathbb{B}}\left(w, \frac{1}{32} d_{D}(w)\right)$.

We may assume that $U \cap \gamma\left[z_{1}, z_{0}\right] \neq \emptyset$. Then by similar discussions as in the proof of Claim 3.7, we get the following Claim. 
Claim 3.13. There exists a sequence of points $\left\{u_{i}\right\}_{i=1}^{p_{2}}$ in $\gamma$, where $p_{2} \geq 2$ is an integer, satisfying the following conditions.

(1) $u_{1}=z_{1}$ or $u_{1}$ is first point in $\gamma\left[z_{1}, z_{0}\right]$ from $z_{1}$ to $z_{0}$ such that $d_{D}\left(u_{1}\right)=$ $64 d_{G}\left(u_{1}\right)$

(2) For each even number $j \in\left\{1,2, \ldots, p_{2}\right\}, d_{D}\left(u_{j}\right) \leq 66 d_{G}\left(u_{j}\right)$ and if $p_{2}$ is an odd number, then $d_{D}\left(u_{p_{2}}\right) \leq 128 d_{G}\left(u_{p_{2}}\right)$;

(3) If $p_{2} \geq 4$, then for each even number $j \in\left\{1,2, \ldots, p_{2}-2\right\}, u_{j+1}$ is the first point in $\gamma\left[u_{j}, z_{2}\right]$ from $u_{j}$ to $z_{2}$ such that $d_{D}\left(u_{j+1}\right)=128 d_{G}\left(u_{j+1}\right)$;

(4) $p_{2}$ is the smallest integer such that $u_{p_{2}}=z_{0}$ or $z_{0} \in \overline{\mathbb{B}}\left(u_{p_{2}-1}, \frac{1}{32} d_{D}\left(u_{p_{2}-1}\right)\right)$.

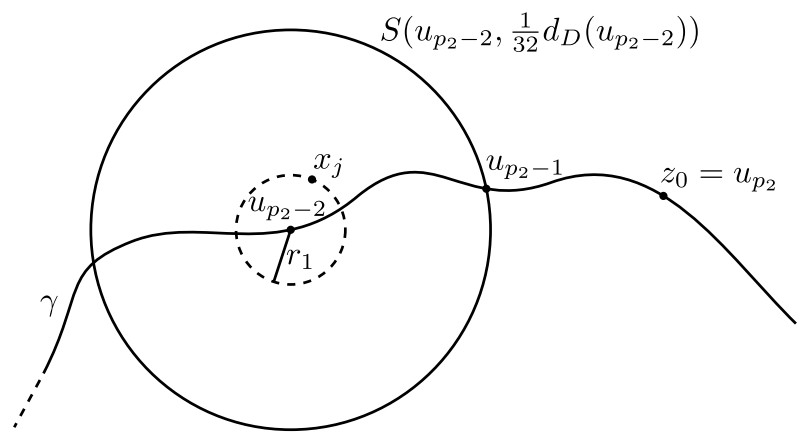

Figure 3. $r_{1}=\frac{1}{128} d_{D}\left(u_{p_{2}-2}\right)$ and $x_{j} \in P_{D}$

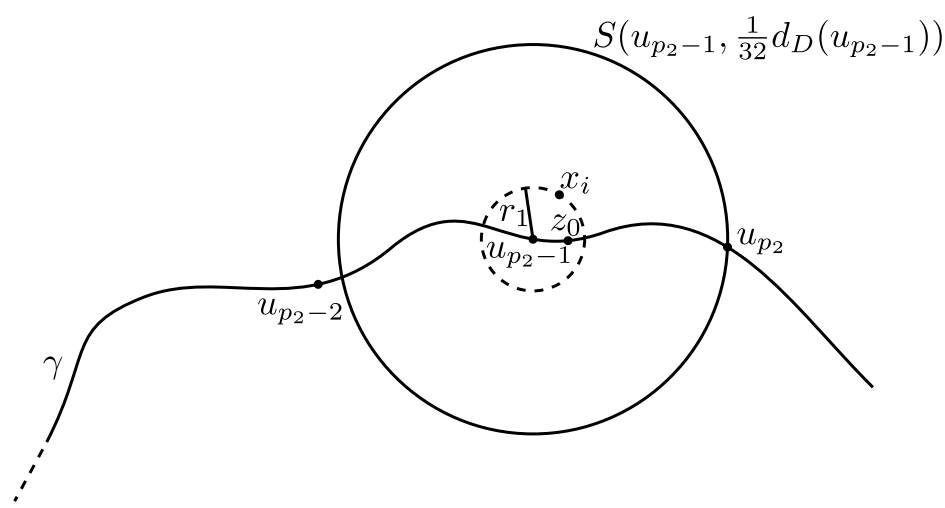

Figure 4. $r_{1}=\frac{1}{128} d_{D}\left(u_{p_{2}-1}\right)$ and $x_{i} \in P_{D}$

We note that there are two possibilities for $u_{p_{2}}$ (see Figures 3 and 4 ): One is $u_{p_{2}}=z_{0}$ and for all $w \in \gamma\left[u_{p_{2}-1}, u_{p_{2}}\right], d_{D}(w) \leq 128 d_{G}(w)$; and the other is $u_{p_{2}} \in \overline{\mathbb{B}}\left(u_{p_{2}-1}, \frac{1}{32} d_{D}\left(u_{p_{2}-1}\right)\right) \cap \gamma\left[z_{0}, z_{2}\right]$. No matter in which case, the proof is similar. So, in the following, we assume that $u_{p_{2}} \in \overline{\mathbb{B}}\left(u_{p_{2}-1}, \frac{1}{32} d_{D}\left(u_{p_{2}-1}\right)\right) \cap \gamma\left[z_{0}, z_{2}\right]$. Then $p_{2}$ is an even number, and by Claim 3.13, we note that

$$
d_{D}\left(u_{p_{2}}\right) \leq 66 d_{G}\left(u_{p_{2}}\right) .
$$

We assume that $U \cap \gamma\left[z_{2}, u_{p_{2}}\right] \neq \emptyset$. Then by similar discussions as in the proof of Claim 3.7, we also get the following claim. 
Claim 3.15. There exists a sequence of points $\left\{v_{i}\right\}_{i=1}^{p_{3}}$ in $\gamma\left[z_{2}, u_{p_{2}}\right]$, where $p_{3} \geq 2$ is an integer, satisfying the following conditions.

(1) $v_{1}=z_{2}$ or $v_{1}$ is the first point in $\gamma\left[z_{2}, u_{p_{2}}\right]$ from $z_{2}$ to $u_{p_{2}}$ such that $d_{D}\left(v_{1}\right)=$ $64 d_{G}\left(v_{1}\right)$

(2) For each even number $j \in\left\{1,2, \ldots, p_{3}\right\}, d_{D}\left(v_{j}\right) \leq 66 d_{G}\left(v_{j}\right)$ and $d_{D}\left(u_{p_{3}}\right) \leq$ $66 d_{G}\left(u_{p_{3}}\right)$

(3) If $p_{3} \geq 4$, then for each even number $j \in\left\{1,2, \ldots, p_{3}-2\right\}, v_{j+1}$ is the first point in $\gamma\left[v_{j}, u_{p_{2}}\right]$ from $v_{j}$ to $u_{p_{2}}$ such that $d_{D}\left(v_{j+1}\right)=128 d_{G}\left(v_{j+1}\right)$;

(4) $p_{3}$ is the smallest integer such that $u_{p_{2}}=v_{p_{3}}$ or $u_{p_{2}} \in \overline{\mathbb{B}}\left(v_{p_{3}-1}, \frac{1}{32} d_{D}\left(v_{p_{3}-1}\right)\right)$.

We note that what we consider here is the case when $p_{2}$ is an even number, then by using the similar method as in the discussion of Case 3.3, together with Lemma 2.15. Claims 3.13 and 3.15 , we construct an arc $\alpha_{5}=\alpha_{5}^{\prime} \cup \alpha_{5}^{\prime \prime}$ in $G$ such that $\alpha_{5}^{\prime}=\gamma\left[z_{1}, u_{1}\right] \cup \beta_{1,1}\left[u_{1}, u_{2}\right] \cup \ldots \cup \beta_{1, i}\left[u_{2 i-1}, u_{2 i}\right] \cup \gamma\left[u_{2 i}, u_{2 i+1}\right] \cup \ldots \cup \beta_{1, \frac{p_{2}}{2}}\left[u_{p_{2}-1}, u_{p_{2}}\right]$ and if $p_{3}$ is an odd number, then

$$
\alpha_{5}^{\prime \prime}=\gamma\left[z_{2}, v_{1}\right] \cup \beta_{2,1}\left[v_{1}, v_{2}\right] \cup \ldots \cup \beta_{2, i}\left[v_{2 i-1}, v_{2 i}\right] \cup \gamma\left[v_{2 i}, v_{2 i+1}\right] \cup \ldots \cup \gamma\left[v_{p_{3}-1}, u_{p_{2}}\right] \text {; }
$$

if $p_{3}$ is en even number, then

$\alpha_{5}^{\prime \prime}=\gamma\left[z_{2}, v_{1}\right] \cup \beta_{2,1}\left[v_{1}, v_{2}\right] \cup \ldots \cup \beta_{2, i}\left[v_{2 i-1}, v_{2 i}\right] \cup \gamma\left[v_{2 i}, v_{2 i+1}\right] \cup \ldots \cup \beta_{2, \frac{p_{3}}{2}}\left[v_{p_{3}-1}, u_{p_{2}}\right]$,

where $\beta_{1, i}\left[u_{2 i-1}, u_{2 i}\right]$ and $\beta_{2, i}\left[v_{2 i-1}, v_{2 i}\right]$ are $c_{2}$-uniform arcs in $G$.

Obviously,

$$
\ell\left(\alpha_{5}\right) \leq c_{2} \ell(\gamma)
$$

Moreover, if $\gamma$ is a $c$-cone arc, we prove that for all $w \in \alpha_{5}$,

$$
\min \left\{\ell\left(\alpha_{5}\left[z_{1}, w\right]\right), \ell\left(\alpha_{5}\left[w, z_{2}\right]\right)\right\} \leq 8 c_{2}^{2}(32 c+1) d_{G}(w) .
$$

To show this, we only need to consider the case $w \in \alpha_{5}^{\prime}$.

If $w \in \gamma \cap \alpha_{5}^{\prime}$, then

$$
\ell\left(\alpha_{5}\left[z_{1}, w\right]\right) \leq c_{2} \ell\left(\gamma\left[z_{1}, w\right]\right) \leq 128 c c_{2} d_{G}(w) .
$$

If $w \in \beta_{1,1}\left[u_{1}, u_{2}\right] \cup \ldots \cup \beta_{1, \frac{p_{2}}{2}}\left[u_{p_{2}-1}, u_{p_{2}}\right]$, then there exists some $i \in\left\{1,2, \ldots, \frac{p_{2}}{2}\right\}$ such that $w \in \beta_{1, i}$. Hence by Lemma 2.16. Claim 3.13, (3.11) and (3.14), we have for all $w \in \beta_{1, i}\left[u_{2 i-1}, u_{2 i}\right]$,

$$
\begin{aligned}
\ell\left(\alpha_{5}\left[z_{1}, w\right]\right) & \leq c_{2} \ell\left(\gamma\left[z_{1}, u_{2 i-1}\right]\right)+\ell\left(\beta_{1, i}\left[u_{2 i-1}, w\right]\right) \\
& \leq 128 c c_{2} d_{G}\left(u_{2 i-1}\right)+c_{2}\left|u_{2 i-1}-u_{2 i}\right| \\
& \leq 4 c_{2}(32 c+1) d_{G}\left(u_{2 i-1}\right) \leq 8 c_{2}^{2}(32 c+1) d_{G}(w) .
\end{aligned}
$$

Hence, Lemma 3.1 is follows from Cases $3.2,3.3$ and 3.12 .

Theorem 3.16. A domain $D \subset E$ is a c-John domain if and only if $G=D \backslash P_{D}$ is a $c_{1}$-John domain, where $c \geq 1$ and $c_{1} \geq 1$ depend only on each other. 
Proof. Necessity: Let $D$ be a $c$-John domain. For given $z_{1}, z_{2} \in G$, there is a $c$ cone arc $\gamma$ in $D$ joining $z_{1}$ and $z_{2}$. By Lemma 3.1, we know that there exists a $\left(2^{18} c c_{2}^{3}+660 c c_{2}^{2}\right)$-cone arc in $G$ joining $z_{1}$ and $z_{2}$. Then $G$ is a John domain.

Sufficiency: Let $c=\frac{65}{63} c_{1}$. For each $z_{1}, z_{2} \in D$, we prove that there exists an arc $\beta \subset D$ joining $z_{1}$ and $z_{2}$ such that

$$
\min \left\{\ell\left(\beta\left[z_{1}, w\right]\right), \ell\left(\beta\left[z_{2}, w\right]\right)\right\} \leq c d_{D}(w) \text { for all } w \in \beta .
$$

If $\left|z_{1}-z_{2}\right| \leq \frac{1}{4} \max \left\{d_{D}\left(z_{1}\right), d_{D}\left(z_{2}\right)\right\}$, then let

$$
\beta=\left[z_{1}, z_{2}\right]
$$

and obviously,

$$
\min \left\{\left|z_{1}-w\right|,\left|z_{2}-w\right|\right\} \leq d_{D}(w) \text { for all } w \in\left[z_{1}, z_{2}\right],
$$

which shows that 3.17 holds.

In the following, we assume that $\left|z_{1}-z_{2}\right|>\frac{1}{4} \max \left\{d_{D}\left(z_{1}\right), d_{D}\left(z_{2}\right)\right\}$. If $z_{1}, z_{2} \in G$, then let $\gamma$ be a $c_{1}$-cone arc joining $z_{1}$ and $z_{2}$ in $G$, and take

$$
\beta=\gamma
$$

Then $\beta$ satisfies 3.17 since $G \subset D$.

If $z_{1} \in P_{D}$ but $z_{2} \notin P_{D}$, then let $x \in G$ be such that $\left|z_{1}-x\right|=\frac{1}{64} d_{D}\left(z_{1}\right)$, and $\gamma$ be a $c_{1}$-cone arc joining $x$ and $z_{2}$ in $G$. Take

$$
\beta=\left[z_{1}, x\right] \cup \gamma
$$

If $z_{1} \notin P_{D}$ but $z_{2} \in P_{D}$, then let $y \in G$ be such that $\left|z_{2}-y\right|=\frac{1}{64} d_{D}\left(z_{2}\right)$, and $\gamma$ be a $c_{1}$-cone arc joining $y$ and $z_{1}$ in $G$. Take

$$
\beta=\left[z_{2}, y\right] \cup \gamma
$$

If $z_{1}, z_{2} \in P_{D}$, then let $x \in G$ such that $\left|z_{1}-x\right|=\frac{1}{64} d_{D}\left(z_{1}\right)$ and $y \in G$ such that $\left|z_{2}-y\right|=\frac{1}{64} d_{D}\left(z_{2}\right)$, and $\gamma$ be a $c_{1}$-cone arc joining $x$ and $y$ in $G$. Take

$$
\beta=\left[z_{1}, x\right] \cup \gamma \cup\left[y, z_{2}\right] .
$$

To prove that these three $\operatorname{arcs} \beta$ are cone $\operatorname{arcs}$ in $D$, it is enough to consider the third case where $z_{1} z_{2} \in P_{D}$. In this case, $\beta=\left[z_{1}, x\right] \cup \gamma \cup\left[y, z_{2}\right]$.

Let $z_{0}$ bisect the arclength of $\gamma$. It suffices to prove that for all $w \in \beta\left[z_{1}, z_{0}\right]$,

$$
\ell\left(\beta\left[z_{1}, w\right]\right) \leq \frac{65}{63} c_{1} d_{D}(w) .
$$

On one hand, if $w \in\left[z_{1}, x\right]$, then

$$
\ell\left(\beta\left[z_{1}, w\right]\right)=\left|z_{1}-w\right| \leq \frac{1}{64} d_{D}\left(z_{1}\right) \leq \frac{1}{63} d_{D}(w) .
$$

On the other hand, if $w \in \gamma\left[x, z_{0}\right]$, then Lemma 2.16 shows

$$
d_{D}(w) \geq \frac{1}{2 c_{1}} d_{D}(x),
$$

which, together with (3.18), shows that

$$
\ell\left(\beta\left[z_{1}, w\right]\right)=\left|z_{1}-x\right|+\ell(\gamma[x, w]) \leq \frac{1}{63} d_{D}(x)+c_{1} d_{D}(w) \leq \frac{65}{63} c_{1} d_{D}(w)
$$


Hence (3.17) holds, and so the proof of Theorem 3.16 is complete.

As an application of Theorem 3.16, we show the following result concerning inner uniform domains.

Theorem 3.19. A domain $D \subset E$ is an inner c-uniform domain if and only if $G=D \backslash P_{D}$ is an inner $c_{1}$-uniform domain, where $c \geq 1$ and $c_{1} \geq 1$ depend only on each other.

Proof. We first prove the necessary part of the theorem, that is, if $D$ is an inner $c$-uniform domain, we need to prove that each pair $z_{1}, z_{2} \in G$ can be joined by an inner $c_{1}$-uniform arc in $G$, where $c_{1}=2^{18} c^{2} c_{2}^{3}+660 c_{2}^{2}$, and $c_{2}\left(2 \leq c_{2} \leq 18\right)$ is a constant from Lemma 2.15 .

For $z_{1}, z_{2} \in G$, since $D$ is an inner $c$-uniform domain, then there is an arc $\gamma$ joining $z_{1}$ and $z_{2}$ in $D$ such that for all $w \in \gamma$

$$
\min \left\{\ell\left(\gamma\left[z_{1}, w\right]\right), \ell\left(\gamma\left[z_{2}, w\right]\right)\right\} \leq c d_{D}(w)
$$

and

$$
\ell(\gamma) \leq c \lambda_{D}\left(z_{1}, z_{2}\right)
$$

By Lemma 3.1, we know that there exists an arc $\alpha \subset G$ such that $\alpha$ is a $\left(2^{18} c^{2} c_{2}^{3}+\right.$ $\left.660 c_{2}^{2}\right)$-cone arc in $G$ and $\ell(\alpha) \leq 660 c_{2}^{2} \ell(\gamma)$. Hence

$$
\ell(\alpha) \leq 660 c_{2}^{2} \ell(\gamma) \leq 660 c c_{2}^{2} \lambda_{D}\left(z_{1}, z_{2}\right) \leq 660 c c_{2}^{2} \lambda_{G}\left(z_{1}, z_{2}\right),
$$

which shows that $\alpha$ is the desired arc.

To prove the sufficient part of Theorem 3.19, we need to prove that for each $z_{1}, z_{2} \in D$, there exists an arc $\beta$ joining $z_{1}$ and $z_{2}$ in $D$ such that

$$
\min \left\{\ell\left(\beta\left[z_{1}, w\right]\right), \ell\left(\beta\left[z_{2}, w\right]\right)\right\} \leq\left(1485 c_{1} c_{2}^{2}+\frac{1}{8}\right) d_{D}(w) \text { for all } w \in \beta,
$$

and

$$
\ell(\beta) \leq\left(1485 c_{1} c_{2}^{2}+\frac{1}{8}\right) \lambda_{D}\left(z_{1}, z_{2}\right)
$$

If $\left|z_{1}-z_{2}\right| \leq \frac{1}{4} \max \left\{d_{D}\left(z_{1}\right), d_{D}\left(z_{2}\right)\right\}$, then let

$$
\beta=\left[z_{1}, z_{2}\right] \text {. }
$$

Obviously, $\beta$ satisfies 3.20 and 3.21 .

In the following, we assume that

$$
\left|z_{1}-z_{2}\right|>\frac{1}{4} \max \left\{d_{D}\left(z_{1}\right), d_{D}\left(z_{2}\right)\right\} .
$$

We divide the proof of this case into two parts.

Case 3.23. $z_{1}, z_{2} \in G$.

Since $G$ is an inner $c_{1}$-uniform domain, then there is a $c_{1}$-cone arc $\gamma$ joining $z_{1}$ and $z_{2}$ in $G$ such that

$$
\ell(\gamma) \leq c_{1} \lambda_{G}\left(z_{1}, z_{2}\right)
$$


Obviously, $\gamma$ satisfies 3.20 since $G \subset D$. In order to prove $\gamma$ satisfies (3.21), we let $\alpha$ be an arc joining $z_{1}$ and $z_{2}$ in $D$ with

$$
\ell(\alpha) \leq 2 \lambda_{D}\left(z_{1}, z_{2}\right) .
$$

By Lemma 3.1, we join $z_{1}$ and $z_{2}$ by an arc $\alpha_{1} \subset G$ such that

$$
\ell\left(\alpha_{1}\right) \leq 660 c_{2}^{2} \ell(\alpha)
$$

which, together with 3.24 ) and $(3.25)$, shows that

$$
\ell(\gamma) \leq c_{1} \lambda_{G}\left(z_{1}, z_{2}\right) \leq c_{1} \ell\left(\alpha_{1}\right) \leq 1320 c_{1} c_{2}^{2} \lambda_{D}\left(z_{1}, z_{2}\right) .
$$

Now we take

$$
\beta=\gamma
$$

Obviously, $\beta$ satisfies $(3.20)$ and (3.21).

Case 3.26. $z_{1} \notin G$ or $z_{2} \notin G$.

Without loss of generality, we may assume that $z_{1} \notin G$ and $z_{2} \notin G$, since the proof for the case $z_{1} \in G, z_{2} \notin G$ or $z_{1} \notin G, z_{2} \in G$ is similar. Let $x, y \in G$ be such that

$$
\left|z_{1}-x\right|=\frac{1}{64} d_{D}\left(z_{1}\right), \quad\left|z_{2}-y\right|=\frac{1}{64} d_{D}\left(z_{2}\right),
$$

and let $\gamma$ be an inner $c_{1}$-uniform arc joining $x$ and $y$ in $G$. Take

$$
\beta=\left[z_{1}, x\right] \cup \gamma \cup\left[y, z_{2}\right] .
$$

By Theorem 3.16 and its proof, we know that $\beta$ satisfies 3.20 . It follows from Case 3.23 that

$$
\ell(\gamma) \leq 1320 c_{1} c_{2}^{2} c_{2} \lambda_{D}(x, y)
$$

which, together with 3.22 and $(3.27)$, shows that

$$
\begin{aligned}
\ell\left(\beta\left[z_{1}, z_{2}\right]\right) & =\left|z_{1}-x\right|+\ell(\gamma[x, y])+\left|y-z_{2}\right| \\
& \leq \frac{1}{8}\left|z_{1}-z_{2}\right|+1320 c_{1} c_{2}^{2} \lambda_{D}(x, y) \\
& \leq\left(1485 c_{1} c_{2}^{2}+\frac{1}{8}\right) \lambda_{D}\left(z_{1}, z_{2}\right),
\end{aligned}
$$

from which we see that $\beta$ satisfies (3.21). Hence the proof of Theorem 3.19 is complete.

\section{Stability of $\psi$-John DOMAins}

In [9], the authors considered the $\psi$-John domains which is a generalization of John domains.

Definition 4.1. A domain $D$ is said to be a $\psi$-John domain if $\psi$ is an increasing self-homeomorphism of $[0, \infty]$ and if for some fixed $x_{0} \in D$ and for all $y \in D$, we have

$$
k_{D}\left(x_{0}, y\right) \leq \psi\left(\frac{\left|x_{0}-y\right|}{\min \left\{d_{D}\left(x_{0}\right), d_{D}(y)\right\}}\right) .
$$

The following lemma follows immediately from (2.1). 
Lemma 4.2. If $\psi:[0, \infty] \rightarrow[0, \infty]$ is a homeomorphism such that a domain is a $\psi$-John domain, then $\log (1+t) \leq \psi(t)$ holds for all $t \geq 0$.

By [25, Theorem 2.23], we have the following lemma which is useful for the discussion in the rest of this section.

Lemma 4.3. Suppose that $D \subset E$ is a domain and that $D_{1} \subset D$ is a c-uniform domain. Then for all $x, y \in D_{1}$,

$$
k_{D}(x, y) \leq c_{1} j_{D}(x, y)
$$

with $c_{1}=c_{1}(c) \leq 7 c^{3}$.

From Lemma 2.15 and Lemma 4.3 , we easily get the following corollary.

Corollary 4.4. Suppose that $D \subset E$ is a domain and $G=D \backslash P_{D}$. For $x, y \in D$, if $d_{D}(x)=128 d_{G}(x)$ and $y \in \overline{\mathbb{B}}\left(x, \frac{1}{32} d_{D}(x)\right)$, then $k_{G}(x, y) \leq \mu j_{G}(x, y)$, where $\mu \leq$ $7 \times 18^{3}$ is a constant.

Meanwhile, [29, Lemma 3.7(2)] yields the following corollary.

Corollary 4.5. Suppose that $D \subset E$ is a domain. For $x, y \in D$, if $|x-y| \leq$ $\frac{1}{2} \min \left\{d_{D}(x), d_{D}(y)\right\}$, then $k_{D}(x, y) \leq 2 j_{D}(x, y)$.

Before the statement of our main result in this section, we prove the following two lemmas.

Lemma 4.6. Let $D$ be a domain and $G=D \backslash P_{D}$. For each $x \in D$, there exists some point $w \in \mathbb{S}\left(x, \frac{1}{32} d_{D}(x)\right)$ such that

$$
\frac{1}{48} d_{D}(x)<\frac{1}{33} d_{D}(w) \leq d_{G}(w) \leq \frac{33}{31} d_{D}(w) .
$$

Proof. Let $x \in D$. By Lemma 2.13, there exists at most one point in $P_{D} \cap$ $\mathbb{B}\left(x, \frac{1}{6} d_{D}(x)\right)$. On one hand, if $P_{D} \cap \mathbb{B}\left(x, \frac{1}{6} d_{D}(x)\right)=\emptyset$, let $w \in \mathbb{S}\left(x, \frac{1}{32} d_{D}(x)\right)$. On the other hand, if $P_{D} \cap \mathbb{B}\left(x, \frac{1}{6} d_{D}(x)\right) \neq \emptyset$, then there exists one and only one point $x_{i}$ in $P_{D} \cap \mathbb{B}\left(x, \frac{1}{6} d_{D}(x)\right)$. Let $l$ be a line determined by $x$ and $x_{i}$, and take $w \in l \cap \mathbb{S}\left(x, \frac{1}{32} d_{D}(x)\right)$ such that $d_{G}(w) \geq \frac{1}{32} d_{D}(x)$. Then

and so

$$
d_{D}(w) \leq d_{D}(x)+|w-x| \leq \frac{33}{32} d_{D}(x)
$$

Hence

$$
d_{G}(w) \geq \frac{1}{32} d_{D}(x) \geq \frac{1}{33} d_{D}(w)
$$

and

$$
d_{D}(w) \geq d_{D}(x)-|x-w|=\frac{31}{32} d_{D}(x) \geq \frac{31}{33} d_{G}(w)
$$

The proof is complete.

$$
d_{G}(w) \geq \frac{1}{33} d_{D}(w)>\frac{1}{48} d_{D}(x)
$$

Lemma 4.7. Let $D$ be a domain and $G=D \backslash P_{D}$. For each $x \in D$ and $w \in$ $\mathbb{S}\left(x, \frac{1}{32} d_{D}(x)\right)$, if $d_{D}(x) \geq 128 d_{G}(x)$, then $d_{G}(w) \geq \frac{1}{44} d_{D}(w)$. 
Proof. Observe first that

$$
d_{D}(w) \leq d_{D}(x)+|w-x| \leq \frac{33}{32} d_{D}(x) .
$$

Let $x \in D$. Since $d_{D}(x) \geq 128 d_{G}(x)$, then by Lemma 2.13 , there exists one and only one point, namely $x_{i}$, in $P_{D} \cap \mathbb{B}\left(x, \frac{1}{6} d_{D}(x)\right)$. Hence

$$
d_{G}(w)=\left|w-x_{i}\right| \geq|x-w|-\left|x-x_{i}\right| \geq \frac{3}{128} d_{D}(x) \geq \frac{1}{44} d_{D}(w) .
$$

Thus the proof of the lemma is complete.

For $\psi$-John domains, we get the following stability of $\psi$-John domain.

Theorem 4.8. A domain $D \subset E$ is a $\psi$-John domain if and only if $G=D \backslash P_{D}$ is a $\psi_{1}$-John domain, where $\psi$ and $\psi_{1}$ are homeomorphisms depending only on each other.

Proof. We first prove the necessary part of the theorem. For this, we assume that $D$ is $\psi$-John domain with center $x_{0}$, where $x_{0} \in D$. By Lemma 4.6, there exists some point $w_{0}$ in $\mathbb{S}\left(x_{0}, \frac{1}{32} d_{D}\left(x_{0}\right)\right)$ such that

$$
\frac{1}{48} d_{D}\left(x_{0}\right)<\frac{1}{33} d_{D}\left(w_{0}\right) \leq d_{G}\left(w_{0}\right) \leq \frac{33}{31} d_{D}\left(w_{0}\right)
$$

and

$$
d_{D}\left(w_{0}\right) \leq d_{D}\left(x_{0}\right)+\left|x_{0}-w_{0}\right| \leq \frac{33}{32} d_{D}\left(x_{0}\right) .
$$

We come to prove that there exists some homeomorphism $\psi^{\prime}$ of $[0, \infty)$ such that $G$ is a $\psi^{\prime}$-John domain with center $w_{0}$. That is, we need to find a homeomorphism $\psi^{\prime}$ of $[0, \infty)$ such that for each $y \in G$,

$$
k_{G}\left(w_{0}, y\right) \leq \psi^{\prime}\left(\frac{\left|w_{0}-y\right|}{\min \left\{d_{G}\left(w_{0}\right), d_{G}(y)\right\}}\right) .
$$

For $y \in G$, if $\left|w_{0}-y\right| \leq \frac{1}{2} \max \left\{d_{G}\left(w_{0}\right), d_{G}(y)\right\}$, then Lemmas 4.2 and Corollary 4.5 show that

$$
k_{G}\left(w_{0}, y\right) \leq 2 \log \left(1+\frac{\left|w_{0}-y\right|}{\min \left\{d_{G}\left(w_{0}\right), d_{G}(y)\right\}}\right) \leq 2 \psi\left(\frac{\left|w_{0}-y\right|}{\min \left\{d_{G}\left(w_{0}\right), d_{G}(y)\right\}}\right),
$$

which shows that 4.11 holds with $\psi_{1}(t)=2 \psi(t)$. Hence, in the following, we assume that

$$
\left|w_{0}-y\right|>\frac{1}{2} \max \left\{d_{G}\left(w_{0}\right), d_{G}(y)\right\} .
$$

Let $\gamma$ be a 2-neargeodesic joining $w_{0}$ and $y$ in $D$. We leave the proof for a moment and prove the following claim.

Claim 4.13. There exists a sequence of points $\left\{w_{i}\right\}_{i=0}^{p}$ in $\gamma$, where $p \geq 1$ is an integer, satisfying the following conditions.

(1) For each even number $j \in\{0, \ldots, p-1\}, d_{D}\left(w_{j}\right) \leq 44 d_{G}\left(w_{j}\right)$; 
(2) For each even number $j \in\{0, \ldots, p-1\}, w_{j+1}$ is the first point in $\gamma\left[w_{j}, y\right]$ from $w_{j}$ to $y$ such that $d_{D}\left(w_{j+1}\right)=128 d_{G}\left(w_{j+1}\right)$;

(3) If $p \geq 2$, then for each even number $j \in\{1, \ldots, p\}, w_{j} \in \overline{\mathbb{B}}\left(w_{j-1}, \frac{1}{32} d_{D}\left(w_{j-1}\right)\right)$.

Obviously, by (4.9), we have

$$
d_{D}\left(w_{0}\right) \leq 33 d_{G}\left(w_{0}\right)<44 d_{G}\left(w_{0}\right) .
$$

If for all $w \in \gamma, d_{D}(w)<128 d_{G}(w)$, then let $w_{1}=y$. Then the claim obviously holds with $p=1$. If there exists some point $v_{0} \in \gamma \operatorname{such}$ that $d_{D}\left(v_{0}\right) \geq 128 d_{G}\left(v_{0}\right)$, then by (4.9), there exist a point $w_{1} \in \gamma$ be the first point from $w_{0}$ to $y$ satisfying

$$
d_{D}\left(w_{1}\right)=128 d_{G}\left(w_{1}\right) \text {. }
$$

If $y \in \overline{\mathbb{B}}\left(w_{1}, \frac{1}{32} d_{D}\left(w_{1}\right)\right)$, then the claim holds by letting $w_{2}=y$, and then $p=2$. Otherwise, let $w_{2} \in \gamma \cap \mathbb{S}\left(w_{1}, \frac{1}{32} d_{D}\left(w_{1}\right)\right)$ such that

$$
\gamma\left[w_{2}, y\right] \cap \mathbb{B}\left(w_{1}, \frac{1}{32} d_{D}\left(w_{1}\right)\right)=\emptyset
$$

Then by Lemma 4.7, we have

$$
d_{G}\left(w_{2}\right) \geq \frac{1}{44} d_{D}\left(w_{2}\right)
$$

If for all $w \in \gamma\left[w_{2}, y\right]$,

$$
d_{D}(w) \leq 128 d_{G}(w)
$$

then the claim holds with $w_{3}=y$, and then $p=3$. Otherwise, let $w_{3}$ be the first point in $\gamma\left[w_{2}, y\right]$ from $w_{2}$ to $y$ such that

$$
d_{G}\left(w_{3}\right)=\frac{1}{128} d_{D}\left(w_{3}\right)
$$

By repeating this process for finite steps, we get a sequence $\left\{w_{i}\right\}_{i=0}^{p} \in \gamma$ satisfying the claim, where $p<\frac{M}{\log \frac{33}{32}}$, since for each even number $i \in\{1,2, \ldots, p\}$,

$$
\ell_{k_{D}}\left(\gamma\left[w_{i-1}, w_{i}\right]\right) \geq \log \left(1+\frac{\left|w_{i-1}-w_{i}\right|}{d_{D}\left(w_{i-1}\right)}\right)=\log \frac{33}{32},
$$

and $M=\ell_{k_{D}}\left(\gamma\left[w_{0}, y\right]\right)$. Hence Claim 4.13 holds.

Now, we come back to the proof of the necessary part of the theorem. By Claim 4.13, we know that for each even number $j \in\{0, \ldots, p-1\}$ the following holds: for all $w \in \gamma\left[w_{j}, w_{j+1}\right]$,

Hence

$$
k_{G}\left(w_{j}, w_{j+1}\right) \leq \int_{\gamma\left[w_{j}, w_{j+1}\right]} \frac{|d w|}{d_{G}(w)}<128 \ell_{k_{D}}\left(\gamma\left[w_{j}, w_{j+1}\right]\right) \leq 256 k_{D}\left(w_{j}, w_{j+1}\right) .
$$

By Claim 4.13, we also know that if $p \geq 2$, then for each even number $j \in\{1, \ldots, p\}$, $w_{j} \in \overline{\mathbb{B}}\left(w_{j-1}, \frac{1}{32} d_{D}\left(w_{j-1}\right)\right)$. Hence by Corollary 4.4 and Claim 4.13 , we have

$$
k_{G}\left(w_{j-1}, w_{j}\right) \leq \mu \log \left(1+\frac{\left|w_{j-1}-w_{j}\right|}{\min \left\{d_{G}\left(w_{j-1}\right), d_{G}\left(w_{j}\right)\right\}}\right) \leq 128 \mu k_{D}\left(w_{j-1}, w_{j}\right),
$$

where $\mu$ is the constant from Corollary 4.4 . 
Now we divided the rest part of proof into two cases.

Case 4.16. $d_{G}(y) \geq \frac{1}{128} d_{D}(y)$.

By (4.9) and 4.12), we have

$$
\left|x_{0}-w_{0}\right|=\frac{1}{32} d_{D}\left(x_{0}\right)<\frac{3}{2} d_{G}\left(w_{0}\right) \leq 3\left|y-w_{0}\right|
$$

and

$$
\left|x_{0}-y\right| \leq\left|x_{0}-w_{0}\right|+\left|y-w_{0}\right| \leq 4\left|y-w_{0}\right|,
$$

which, together with 4.9), 4.10), Claim 4.13, (4.14) and 4.15), shows that

$$
\begin{aligned}
k_{G}\left(w_{0}, y\right) & \leq \sum_{i=0}^{p-1} k_{G}\left(w_{i}, w_{i+1}\right) \leq 256 \mu \sum_{i=0}^{p-1} k_{D}\left(w_{i}, w_{i+1}\right) \\
& \leq 512 \mu k_{D}\left(w_{0}, y\right) \leq 512 \mu\left(k_{D}\left(x_{0}, w_{0}\right)+k_{D}\left(x_{0}, y\right)\right) \\
& \leq 512 \mu \psi\left(\frac{\left|x_{0}-w_{0}\right|}{\min \left\{d_{D}\left(x_{0}\right), d_{D}\left(w_{0}\right)\right\}}\right) \\
& +512 \mu \psi\left(\frac{\left|x_{0}-y\right|}{\min \left\{d_{D}\left(x_{0}\right), d_{D}(y)\right\}}\right) \\
& \leq \psi_{2}\left(\frac{\left|y-w_{0}\right|}{\min \left\{d_{G}(y), d_{G}\left(w_{0}\right)\right\}}\right)
\end{aligned}
$$

where $\psi_{2}(t)=1024 \mu \psi(8 t)$.

Case 4.20. $d_{G}(y)<\frac{1}{128} d_{D}(y)$.

In this case, by Claim 4.13, we see that $p$ must be an even number and $p \geq 2$, and then $y \in \overline{\mathbb{B}}\left(w_{p-1}, \frac{1}{32} d_{D}\left(w_{p-1}\right)\right)$. If $w_{0} \in \overline{\mathbb{B}}\left(w_{p-1}, \frac{1}{32} d_{D}\left(w_{p-1}\right)\right)$, then by Corollary 4.4 and Claim 4.13 , we get

$$
k_{G}\left(w_{0}, y\right) \leq \mu \log \left(1+\frac{\left|w_{0}-y\right|}{\min \left\{d_{G}\left(w_{0}\right), d_{G}(y)\right\}}\right) \leq \psi_{3}\left(\frac{\left|w_{0}-y\right|}{\min \left\{d_{G}\left(w_{0}\right), d_{G}(y)\right\}}\right),
$$

where $\psi_{3}(t)=\mu \psi(t)$.

If $w_{0} \notin \overline{\mathbb{B}}\left(w_{p-1}, \frac{1}{32} d_{D}\left(w_{p-1}\right)\right)$, then by 4.18,

$$
d_{G}(y)<\frac{1}{128} d_{D}(y) \leq \frac{1}{128}\left(d_{D}\left(w_{p-1}\right)+\left|w_{p-1}-y\right|\right)<\frac{1}{64} d_{D}\left(w_{p-1}\right)
$$

and

$$
\left|w_{p-1}-x_{0}\right| \leq\left|w_{p-1}-y\right|+\left|x_{0}-y\right|<5\left|y-w_{0}\right|
$$


which, together with Lemma 4.2, (4.9), 4.14, 4.15) and (4.17), shows that

$$
\begin{aligned}
k_{G}\left(w_{0}, y\right) & \leq 256 \mu \sum_{i=0}^{p-2} k_{D}\left(w_{i}, w_{i+1}\right)+k_{G}\left(w_{p-1}, y\right) \\
& \leq 512 \mu k_{D}\left(w_{0}, w_{p-1}\right)+\mu \log \left(1+\frac{\left|w_{p-1}-y\right|}{\min \left\{d_{G}\left(w_{p-1}\right), d_{G}(y)\right\}}\right) \\
& \leq 512 \mu\left(k_{D}\left(x_{0}, w_{0}\right)+k_{D}\left(x_{0}, w_{p-1}\right)\right)+\mu \log \left(1+\frac{\left|w_{p-1}-y\right|}{\min \left\{d_{G}\left(w_{p-1}\right), d_{G}(y)\right\}}\right) \\
& \leq 512 \mu\left(\psi\left(\frac{\left|x_{0}-w_{0}\right|}{\min \left\{d_{D}\left(x_{0}\right), d_{D}\left(w_{0}\right)\right\}}\right)+\psi\left(\frac{\left|x_{0}-w_{p-1}\right|}{\min \left\{d_{D}\left(x_{0}\right), d_{D}\left(w_{p-1}\right)\right\}}\right)\right) \\
& +\mu \log \left(1+\frac{\left|w_{p-1}-y\right|}{\min \left\{d_{G}\left(w_{p-1}\right), d_{G}(y)\right\}}\right) \\
& \leq \psi_{1}\left(\frac{\left|w_{0}-y\right|}{\min \left\{d_{G}\left(w_{0}\right), d_{G}(y)\right\}}\right),
\end{aligned}
$$

where $\psi_{4}(t)=1025 \mu \psi(8 t)$. Hence (4.11) holds with $\psi^{\prime}(t)=1025 \mu \psi(8 t)$.

Now we are going to prove the sufficient part of Theorem 4.8 .

Assume that $G$ is $\psi_{1}$-John domain with center $z_{0}$, where $z_{0} \in G$. By Lemma 4.6 , there exists some point $y_{0}$ in $\mathbb{S}\left(z_{0}, \frac{1}{32} d_{D}\left(z_{0}\right)\right)$ such that

$$
\frac{1}{48} d_{D}\left(z_{0}\right)<\frac{1}{33} d_{D}\left(y_{0}\right) \leq d_{G}\left(y_{0}\right) \leq \frac{33}{31} d_{D}\left(y_{0}\right)
$$

We show that there exists a homeomorphism $\psi$ of $[0, \infty)$ such that $D$ is a $\psi$-John domain with center $y_{0}$. By the necessary part of the theorem, we know that $G_{1}=$ $G \backslash\left\{z_{0}\right\}$ is a $\psi^{\prime}$-John domain with center $y_{0}$, where $\psi^{\prime}(t)=1025 \mu \psi_{1}(8 t)$.

For $y \in D$, if $\left|y_{0}-y\right| \leq \frac{1}{2} \max \left\{d_{D}\left(y_{0}\right), d_{D}(y)\right\}$, then Lemmas 4.2 and Corollary 4.5 show that

$$
k_{D}\left(y_{0}, y\right) \leq 2 \log \left(1+\frac{\left|y_{0}-y\right|}{\min \left\{d_{D}\left(y_{0}\right), d_{D}(y)\right\}}\right) \leq \psi_{1}^{\prime}\left(\frac{\left|y_{0}-y\right|}{\min \left\{d_{D}\left(y_{0}\right), d_{D}(y)\right\}}\right),
$$

where $\psi_{1}^{\prime}(t)=2 \psi_{1}(t)$ and 2 is from Corollary 4.5 . In the following, we assume that

$$
\left|y_{0}-y\right| \geq \frac{1}{2} \max \left\{d_{D}(y), d_{D}\left(y_{0}\right)\right\}
$$

If $d_{D}(y) \leq 62 d_{G}(y)$, then by 4.21),

$$
\left|y-z_{0}\right| \geq\left|y-y_{0}\right|-\left|y_{0}-z_{0}\right| \geq \frac{1}{128} d_{D}\left(z_{0}\right) .
$$

Now we claim that

$$
d_{G}(y) \leq 129 d_{G_{1}}(y)
$$

In fact, if $d_{G}(y)=d_{G_{1}}(y)$, then the above inequality is obvious. If $d_{G}(y)>d_{G_{1}}(y)$, then $d_{G_{1}}(y)=\left|y-z_{0}\right|$. Hence

$$
d_{G}(y) \leq d_{D}\left(z_{0}\right)+\left|z_{0}-y\right| \leq 129 d_{G_{1}}(y),
$$

which shows 4.23). 
Similarly, we have

$$
d_{G}\left(y_{0}\right) \leq 129 d_{G_{1}}\left(y_{0}\right) .
$$

Hence (4.21) and (4.23) yields

$$
k_{D}\left(y_{0}, y\right) \leq k_{G_{1}}\left(y_{0}, y\right) \leq \psi^{\prime}\left(\frac{\left|y_{0}-y\right|}{\min \left\{d_{G_{1}}\left(y_{0}\right), d_{G_{1}}(y)\right\}}\right) \leq \psi_{2}^{\prime}\left(\frac{\left|y_{0}-y\right|}{\min \left\{d_{D}\left(y_{0}\right), d_{D}(y)\right\}}\right),
$$

where $\psi_{2}^{\prime}(t)=1025 \mu \psi_{1}\left(2^{15} t\right)$.

If $d_{D}(y) \geq 62 d_{G}(y)$, then for $y_{1} \in \mathbb{S}\left(y, \frac{1}{16} d_{D}(y)\right)$, Lemma 2.13 implies

$$
d_{D}\left(y_{1}\right) \leq d_{D}(y)+\left|y_{1}-y\right| \leq 32 d_{G}\left(y_{1}\right) .
$$

Hence, a similar proof as to 4.23 leads to

$$
d_{G}\left(y_{1}\right) \leq 129 d_{G_{1}}\left(y_{1}\right),
$$

which, together with Corollary 4.5, (4.21), 4.22), (4.24) and 4.25), shows that

$$
\begin{aligned}
k_{D}\left(y_{0}, y\right) & \leq k_{G_{1}}\left(y_{0}, y_{1}\right)+k_{D}\left(y_{1}, y\right) \\
& \leq \psi^{\prime}\left(\frac{\left|y_{0}-y_{1}\right|}{\min \left\{d_{G_{1}}\left(y_{0}\right), d_{G_{1}}\left(y_{1}\right)\right\}}\right)+2 \log \left(1+\frac{\left|y_{1}-y\right|}{\min \left\{d_{D}\left(y_{1}\right), d_{D}(y)\right\}}\right) \\
& \leq \psi_{3}^{\prime}\left(\frac{\left|y_{0}-y\right|}{\min \left\{d_{D}\left(y_{0}\right), d_{D}(y)\right\}}\right),
\end{aligned}
$$

where $\psi_{3}^{\prime}(t)=1025(\mu+2) \psi_{1}\left(2^{15} t\right)$, and $\mu$ is the constant from Corollary 4.4. By letting $\psi(t)=1025(\mu+2) \psi_{1}\left(2^{15} t\right)$, we get the sufficient part of the theorem. Hence the proof of the theorem is complete.

Remark 4.26. Let $\psi:[0, \infty] \rightarrow[0, \infty]$ be a homeomorphism and $c, \lambda_{1}, \lambda_{2}$ be positive constants. We define the following class:

$$
\Psi_{\lambda_{1}, \lambda_{2}}=\left\{\psi: \lambda_{1} \leq \frac{\psi(c t)}{\psi(t)} \leq \lambda_{2}\right\}
$$

The proof of Theorem 4.8 yields the following quantitative statement: $\psi_{1}(t)=$ $b_{1} \psi\left(b_{2} t\right)$ and $\psi_{2}(t)=b_{3} \psi\left(b_{4} t\right)$ for some positive constants $b_{j}$. Thus we see that if $D$ is a $\psi$-John domain with $\psi \in \Psi_{\lambda_{1}, \lambda_{2}}$, then $D \backslash P_{D}$ is a $\psi_{1}$-John domain with $\psi_{1} \in \Psi_{\lambda_{1}, \lambda_{2}}$. The converse implication also holds.

\section{REFERENCES}

1. G. D. Anderson, M. K. Vamanamurthy and M. Vuorinen, Dimension-free quasiconformal distortion in $n$-space. Trans. Amer. Math. Soc., 297(1986), 687-706.

2. M. Bonk, J. Heinonen And P. Koskela, Uniformizing Gromov hyperbolic domains, Asterisque 270(2001), 1-99.

3. O. J. Broch, Geometry of John disks, Ph. D. Thesis, NTNU, 2004.

4. F. W. Gehring, Uniform domains and the ubiquitous quasidisks, Jahresber. Deutsch. Math. Verein, 89(1987), 88-103.

5. F. W. Gehring And B. P. PAlka, Quasiconformally homogeneous domains, J. Analyse Math., 30(1976), 172-199.

6. F. W. Gehring And B. G. Osgood, Uniform domains and the quasi-hyperbolic metric, $J$. Analyse Math., 36(1979), 50-74. 
7. P. Hästö, S. Ponnusamy and S. K. Sahoo, Inequalities and geometry of the Apollonian and related metrics, Rev. Roumaine Math. Pures Appl. 51(2006), 433-452.

8. P. Hästö, Z. Ibragimov, D. Minda, S. Ponnusamy and S. Sahoo, Isometries of some hyperbolic-type path metrics, and the hyperbolic medial axis. In the tradition of Ahlfors-Bers. IV, Edited by Dick Canary, Jane Gilman, Juha Heinonen and Howard Masur. 63-74, Contemp. Math., 432, Amer. Math. Soc., Providence, RI, 2007.

9. D. A. Herron and M. Vuorinen, Positive harmonic functions in uniform and admissible domains, Analysis, 8(1988), no. 1-2, 187-206, MR0954466.

10. M. Huang, S. Ponnusamy and X. Wang, Decomposition and removability properties of John domains, Proc. Indian Acad. Sci. (Math. Sci.), 118(2008),357-370.

11. M. Huang, S. Ponnusamy, H. Wang and X. Wang, A cosine inequality in hyperbolic geometry, App. Math. Letters, 23(2010),887-891.

12. F. John, Rotation and strain. Comm. Pure Appl. Math. 14(1961) 391-413.

13. R. KLÉN, Local convexity properties of quasihyperbolic balls in punctured space, J. Math. Anal. Appl., 342(2008), 192-201.

14. R. Klén, Y. Li, S. K. SAhoo And M. Vuorinen, On the stability of $\varphi$-uniform domains, Monatshefte für Mathematik, accept, arXiv: 0812.4369v5 [math.MG].

15. R. Klén, A. RAsila, And J. TAlPonen, Quasihyperbolic geometry in euclidean and Banach spaces, Proc. ICM2010 Satellite Conf. International Workshop on Harmonic and Quasiconformal Mappings (HMQ2010), eds. D. Minda, S. Ponnusamy, N. Shanmugalingam, J. Analysis 18 (2011),261-278, arXiv:1104.3745v1 [math.CV].

16. Y. Li AND X. WANG, Unions of John domains and uniform domains in real normed vector spaces, Ann. Acad. Sci. Fenn. Ser. Math., 35(2010), 627-632.

17. O. Martio, Definitions of uniform domains, Ann. Acad. Sci. Fenn. Ser. A I Math., 5(1980), 197-205.

18. O. Martio and J. Sarvas, Injectivity theorems in plane and space, Ann. Acad. Sci. Fenn. Ser. A I Math., 4(1978), 383-401.

19. R. NÄKKI AND J. VÄISÄLÄ, John disks, Expo. Math., 9(1991), 3-43.

20. A. Rasila and J. Talponen, Convexity properties of quasihyperbolic balls on Banach spaces, Ann. Acad. Sci. Fenn. 37 (2012), 215-228, arXiv:1007.3197v1 [math. CV].

21. J. VÄısÄLÄ, Uniform domains, Tohoku Math. J., 40(1988), 101-118.

22. J. VÄIsÄLÄ, Free quasiconformality in Banach spaces. I, Ann. Acad. Sci. Fenn. Ser. A I Math., 15(1990), 355-379.

23. J. VÄIsÄLÄ, Broken tubes in Hilbert spaces. Analysis (Munich) 24(2004), no. 3, 227-238.

24. J. VÄIsÄLÄ, Free quasiconformality in Banach spaces. II, Ann. Acad. Sci. Fenn. Ser. A I Math., 16(1991), 255-310.

25. J. VÄIsÄLÄ, Relatively and inner uniform domains, Conformal Geom. Dyn., 2(1998), 56-88.

26. J. VÄIsÄLÄ, The free quasiworld: freely quasiconformal and related maps in Banach spaces. Quasiconformal geometry and dynamics (Lublin 1996), Banach Center Publications, Vol. 48, Polish Academy of Science, Warsaw, ed. by Bogdan Bojarski, Julian Lawrynowicz, Olli Martio, Matti Vuorinen and Józef Zając, 1999, 55-118.

27. J. VÄIsÄLÄ, Quasihyperbolic geometry of planar domains. Ann. Acad. Sci. Fenn. Math. 34(2009), 447-473.

28. M. Vuorinen, Capacity densities and angular limits of quasiregular mappings. Trans. Amer. Math. Soc. 263(1981), 343-354.

29. M. Vuorinen, Conformal Geometry and Quasiregular Mappings, Lecture Notes in Mathematics 1319, Springer-Verlag, Berlin-Heidelberg-New York, 1988. 
Yaxiang. Li, College of Science, Central South University of Forestry and Technology, Changsha, Hunan 410004, People's Republic of China

E-mail address: yaxiangli@163.com

Matti. Vuorinen, Department of Mathematics and Statistics, University of Turku, FIN-20014 TURKU, Finland

E-mail address: vuorinen@utu.fi

Xiantao. Wang, Department of Mathematics, Hunan Normal University, ChangSha, Hunan 410081, People's Republic of China

E-mail address: xtwang@hunnu.edu.cn 